\title{
The Research based on u-Blox GPS for Material Yard Vehicle Monitor
}

\author{
Li Xue-fen \\ Department of control, Wuxi institute of technology, WuXi, JiangSu, China \\ e-mail: tree0816@163.com
}

Keyword: GPS, material yard, ZigBee, monitor

\begin{abstract}
For the vehicle management require of the large materials yard, this paper researched the monitor problem based on u- Blox GPS. Designed the GPS applicable unit, researched the Setting Method of LEA-4H and the extract skill of GPS Protocol data. Researched the Grid-based map technology to mark, zoom, translate the map and match the vehicle track. Propose the "same side judgment" to check the vehicle whether in the yard only by GPS location. Fig.4 shows how to judge the non-convex polygon yard by divide it into convex. The correctness and stability of the research result are proved by the reliable operation of an unmanned iron ore yard vehicle system for years. The researches provide good reference for the design of the monitor and manage system in other material yard and fleet.
\end{abstract}

\section{Introduction}

In the large cargoes or material stack yard (short for materials yard) of colliery or steel works, the primary task of the managing is avoiding the collision between stacker crane and vehicles to ensure safe. It is important to get the vehicle's accurate location. Because of low-cost and locating accurately, GPS (Global Positioning System) receiver is widely applied in airplanes, ships and cars.

Based on new u-Blox GPS receiver, combined with actual needs of materials yard managing, this paper research how to monitor and manage the vehicles in yard. Firstly, GPS applicable unit was designed based on u-Blox GPS module. Then, study the coordinate transformation method of GPS and analysis the precision. Finally, combined the GPS location, the detection method for vehicle in out the yard is researched.

\section{Design of u-Blox GPS applicable unit}

u-Blox LEA-4H GPS. As the user device of GPS navigation-satellite, GPS receiver is terminal equipment to realize the Location and Navigation of GPS Satellite [1]. GPS receivers can be divided into time-service type, Surveying type and navigation-based type [2]. Navigation-based receiver is mainly used in moving vehicles.

As a Switzerland Company engaged in GPS receiver's research and manufacturing, u-Blox provides an excellent dynamic navigation performance in limited sky view area by its unique ANTARIS GPS [3] module. For example, it can provide high sensitivity at weak signal without reducing the accuracy and support DGPS and multi-SBAS (WAAS and EGNOS) system in the urban canyons. Ensure fast boot times with 16 parallel channels and 8192 search position. With low-power and power-saving model, it also applies to handset and battery driven equipment. Among multi-series of u-Blox such as LEA and TIM, the LEA-4H GPS receiver is navigation-based type with 16 channels operated in the L1 band with C/A code and has some advantages such as low-energy, start-up fast, good dynamic performance and easy integration in other circuits. With $-158 \mathrm{dBm}$ tracking sensitivity, it can be used in more area with more small or hidden antenna. It is suitable for industry application with operating temperature range between -40 and $85^{\circ} \mathrm{C}$. So, LEA-4H GPS module is selected in this paper.

Hardware design. The LEA-4H GPS structural principle is shown in Fig.1. As we can see from the Fig.1, the basic design of application only needs power and GPS antenna to communicate with external devices through USP or UART port and output the location information. 

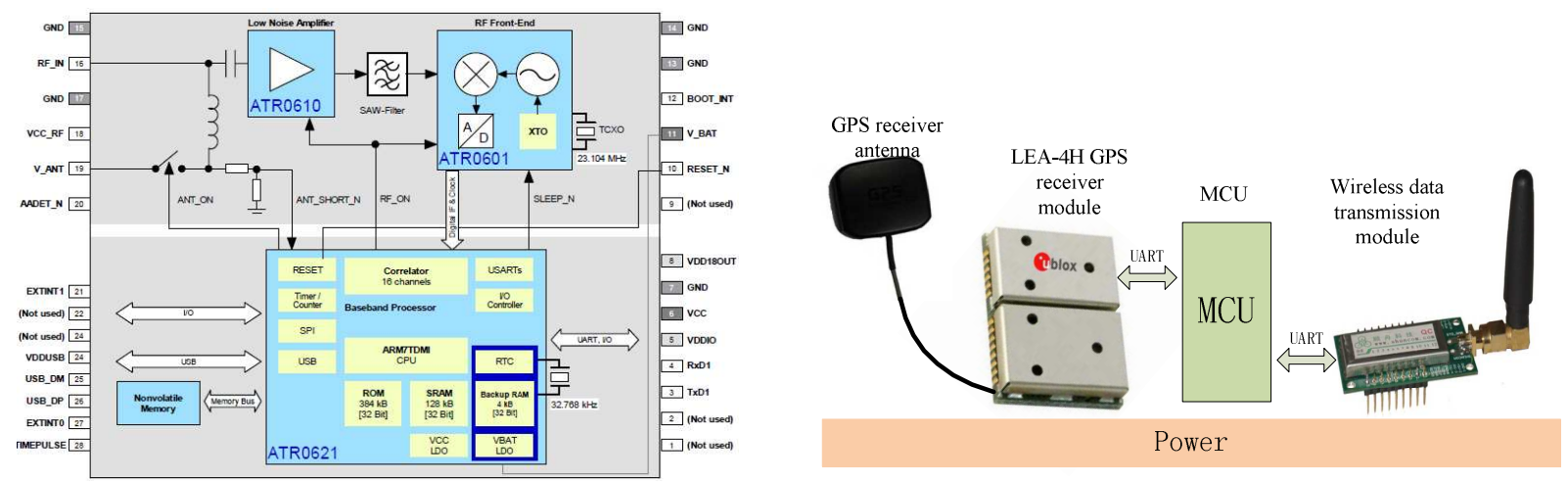

Fig. 1 LEA-4H module structural

Fig. 2 Hardware components of GPS application unit

Wireless data transmission module is used to send the vehicle location message to manage center. ZigBee is a cheap, low-power short-range wireless network communication technology [4]. Its transfer rat is $10 \mathrm{kB} \sim 250 \mathrm{kB} / \mathrm{s}$. In standby mode, two regular AA batteries can last 2 months. The node in ZigBee can up to 65000 and the messages like bees, can look for the best path to the receiver. So, the SZ05 wireless data transmission module based on ZigBee is adopted in this paper.

According to the above research, the hardware design for GPS wireless application unit is presented and shown in Fig.2. MCU sets the GPS and ZigBee wireless data transmission module through UART port, parses and extracts the GPS location information, then sends out it through another UART port.

Setting the LEA-4H GPS module. In theory, this receiver can sent signal directly without designed interface circuit. Its output protocol is under NMEA0183 standard protocol which has ten kinds such as GPGGA [5].

In practice, with $1 \mathrm{HZ}$ in 9.6kbps baud, the receiver sends the GGA, GLL, GSA, GSV, RMC, VTG and ZDA protocol data in default state after cold boot. But there are four problems: 1) to give full play, update rate should be set to $4 \mathrm{HZ}$ by software. 2) Each transmission sends more than 560 bytes in eight kinds protocols including so many overlap or no-relate to practice information. 3) With $4 \mathrm{HZ}$ update rate, the bps is more than 2240 bytes exceeding the default communication bandwidth and losing the data. 4) MCU will process lots of redundant information and real-time of location will be effected by default.

u-Blox developed exclusive NMEA protocol for the users such as PUBX00 which including all kinds of cared information. So, the LEA-4H should be initialized set follow the steps:

1) Set the baud of UART0 in MCU to 9600bps consistent with the GPS default baud.

2) Input related commands in turn to forbid the seven default protocol: ZDA, GSV, RMC, VTG, GGA, GSV and GLL. Delay $0.2 \mathrm{~s}$ after each command to take affect.

3) Input command to permit PUBX00 and delay 2 s to take affect.

4) Input command to change GPS port baud to $38400 \mathrm{bps}$ and delay $2 \mathrm{~s}$ to take affect.

5) Set the baud of UART0 in MCU to $38400 \mathrm{bps}$ consistent with the GPS baud.

6) Input command to set the GPS update rate and navigation frequency to 4HZ. DELAY $1 \mathrm{~S}$ to take affect. 
Take normal process flow after setting GPS module. The commands in steps 2, 3, 4, 6 are shown in table 1.

Table 1 Setup instructions of GPS

\begin{tabular}{|c|c|c|c|}
\hline step & format & Instruction & meaning \\
\hline \multirow{7}{*}{2} & \multirow{9}{*}{ character-oriented } & \$PUBX,40,ZDA, $0,0,0,0 * 44<\mathrm{CR}><\mathrm{LF}>$ & forbit \\
\hline & & \$PUBX,40,GSV, $0,0,0,0 * 59<\mathrm{CR}><\mathrm{LF}>$ & forbit GSV \\
\hline & & \$PUBX,40,RMC, $0,0,0,0 * 47<\mathrm{CR}><\mathrm{LF}>$ & forbit $\mathrm{RMC}$ \\
\hline & & \$PUBX,40,VTG, $0,0,0,0 * 5 \mathrm{E}<\mathrm{CR}><\mathrm{LF}>$ & forbit VTG \\
\hline & & \$PUBX,40,GGA, $0,0,0,0 * 5 \mathrm{~A}<\mathrm{CR}><\mathrm{LF}>$ & forbit GGA \\
\hline & & \$PUBX,40, GSA, $0,0,0,0 * 4 \mathrm{E}<\mathrm{CR}><\mathrm{LF}>$ & forbit GSV \\
\hline & & \$PUBX,40, GLL $, 0,0,0,0 * 5 \mathrm{C}<\mathrm{CR}><\mathrm{LF}>$ & forbit GLL \\
\hline 3 & & \$PUBX,40,00,0,1,0,0*1A $<\mathrm{CR}><\mathrm{LF}>$ & enable PUBX00 \\
\hline 4 & & \$PUBX, 41,1,0003,0003,38400,0*24<CR $><\mathrm{LF}$ & $\begin{array}{l}\text { set the baud of UART0 } \\
\text { in MCU to } 38400 \mathrm{bps}\end{array}$ \\
\hline 6 & bits-oriented & B5 6206080600 FA 0001000000 0F 94 & $\begin{array}{l}\text { set the GPS update rate } \\
\text { and navigation frequency } \\
\text { to } 4 \mathrm{HZ} \text {. }\end{array}$ \\
\hline
\end{tabular}

The extract of PUBX00 Protocol data. After setting LEA-4H, GPS will output PUBX00 Protocol with a $4 \mathrm{HZ}$ update frequency [6]. The PUBX00 Protocol format is:

\$PUBX,00,hhmmss.ss,Latitude,N,Longitude,E,AltRef,NavStat,Hacc,Vacc,SOG,COG,Vvel,ageC ,HDOP,VDOP,TDOP,GU,RU,DR, ${ }^{*} \mathrm{cs}<\mathrm{CR}><\mathrm{LF}>$

In fact, most users only care for finite location information among so many messages contented in the protocol. If exact the concerns to customize a simplified protocol, the data throughput of serial communication and wireless data transmission module will be reduced while reliability improved.

To process the received PUBX00 protocol in MCU, firstly, open a enough receive GPSBuffer(128 Byte), then receives characters begin with " $\$$ " in UATR interrupt service routine and put into GPSBuffer sequentially. Set the receive completed flag (gps frame over) when receive the frame tail $<\mathrm{CR}>$ or $<\mathrm{LF}>$.

Call function to exact GPS data if find gps_frame_over set in mail lop. Reset the flag after the function finished.

The design ideas of exact function as follows:

1) Verify to ensure the soundness and completeness of the received data.

2) According to character-oriented agreement and ordering with comma, exact the relevant character strings and convert them into specified format value by designed GPS simplified protocol.

3) After the conversion, compile the above number into GPS simplified protocol and start the sender.

In that way, all the concerned GPS messages will be sent to monitoring center through wireless data transmission module at $4 \mathrm{HZ}$.

\section{Grid-based map technology}

Map technology is used to reflect the relationship between the moving vehicle and ground location. There are some defects in current vectorization digital map software: 1) Poor open to integration. 2) Update slowly can't match the real situation. 3) Too big scale to reflect the details. So, this paper study the Grid-based map technology which can reflect the real location between vehicle and the ground through marking the map after scanning and rasterizing the common map. 
Marking the map. Marking the map is to establish the relation with GPS location. Specifically: 1) confirm the latitude and longitude of the pixel (usually the map center pixel). 2) confirm the proportion, namely the corresponding increase or reduction of "c" when moving a pixel in the map.

Realize the map marking by confirming the two points of the diagonal. Set the map's resolution to $x_{M} \times y_{M}$, set the latitude and longitude of the two pixels $p 1\left(x_{1}, y_{1}\right), p 2\left(x_{2}, y_{2}\right)$ to $p 1\left(\lambda_{1}, \varphi_{1}\right), p 2\left(\lambda_{2}, \varphi_{2}\right)$. Set the upper-left point as the Device coordinate system's origin $o_{d} x_{d} y_{d}$, positive $x_{d}$ left and $y_{d}$ down. The map's ratio is followed Eq. 1 and the latitude and longitude of the map center is followed Eq. 2 :

$$
\left.\begin{array}{l}
s_{\lambda}=\left(\lambda_{2}-\lambda_{1}\right) /\left(x_{2}-x_{1}\right) \\
s_{\varphi}=\left(\varphi_{2}-\varphi_{1}\right) /\left(y_{2}-y_{1}\right)
\end{array}\right\} .
$$

After marking the map, write the four values of Eq. 1, Eq. 2 in the specific file for procedure calls.

The deformation error in the two-dimensional map is unavoidable while the earth plane is a three-dimensional ellipsoidal surface. It results in the deviation between the actual location and the map's location. Marked by the above methods, the maximum longitude error of the four angular points is followed Eq. 3:

$$
\Delta \lambda= \pm 0.5 s_{\lambda} x_{M}\left(\frac{1}{\cos \varphi_{0}}-\frac{1}{\cos \left(\varphi_{0} \pm 0.5 s_{\varphi} y_{M}\right)}\right) .
$$

For example, if the cars of the materials yard located in shanghai suburb, range for $300 \mathrm{~km} \times$ $300 \mathrm{~km}$ and the map center is situated in 31 degrees north latitude, the maximum longitude error is 2.2578e-9 degree. Less than the GPS location accuracy, it can be neglected.

Zoom and translation. For zooming and translating the map, define the zoon coefficient as $C_{z}$, left and right offset as $\Delta x$, up and down offset as $\Delta y . C_{z}$ initial value $=1$, while $\Delta x$ initial value $=0$ and $\Delta y$ initial value $=0$. Define the location values of the map's upper-left point in the window coordinate system with the origin at the window upper-left point(Y axis is down) as $L_{x}, L_{y}$, define the scope of the map flat out as $x_{D}, y_{D}$.

Update $L_{x}, L_{y}$ and $x_{D}, y_{D}$ by Eq. 5 while $\Delta x$ and (or) $\Delta y$ changed. Update the relevant parameters in Eq. 4 , Eq. 5 in sequence as $C_{z}$ changed then get the zoomed and translated graphics.

$$
\left.\begin{array}{c}
\left.\begin{array}{c}
\Delta x=\Delta x \cdot C_{z} \\
\Delta y_{D}=\Delta y \cdot C_{z}
\end{array}\right\} . \\
x_{D}=x_{M} \cdot C_{z} \\
y_{D}=y_{M} \cdot C_{z} \\
L x=0.5\left(W-x_{D}\right)+\Delta x \\
L y=0.5\left(H-y_{D}\right)+\Delta y
\end{array}\right\} .
$$

The "W", "H" in Eq. 5 means the width and height pixel value in the map display window. 
Match and display the track. To match the vehicle track after zooming and translating the map, the vehicle's latitude and longitude $\lambda_{i}, \varphi_{i}$ should translate to $x_{i}, y_{i}$ through Eq. 6 .

$$
\left.\begin{array}{l}
x_{i}=\left(\lambda_{i}-\lambda_{0}\right) \cdot C z / s_{\lambda}+\Delta x+0.5 \mathrm{~W} \\
y_{i}=\left(\varphi_{i}-\varphi_{0}\right) \cdot C z / s_{\varphi}+\Delta y+0.5 H
\end{array}\right\} .
$$

It's worth pointing out that the sign problem caused by the southern and northern hemispheres is needless to consider in computing $y_{i}$ because it has been brought into Eq. 1 automatically. User can form the dynamic track matched with the map actual location by lining the vehicle location in time order.

\section{In out check method based on GPS}

The main work of yard manage is monitoring the vehicle to ensure the safe. The key problem is how to judge whether the vehicle in the yard.

Usually, the yard shape is regular, rectangular. Show $\mathrm{n}$ in Fig. 3, considered with the counter-clockwise vertices $\boldsymbol{P}_{1}, \boldsymbol{P}_{2}$ to $\boldsymbol{P}_{n}$ of the convex polygon yard, the problem is how to judge the moving point " $\mathrm{M}$ " (the vehicle) in or out of the yard.

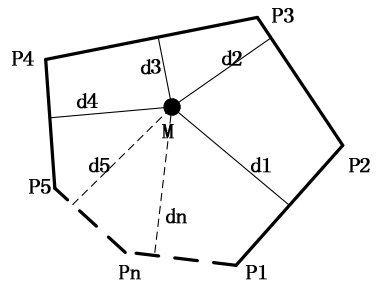

Fig. 3 Judge method for convex polygon

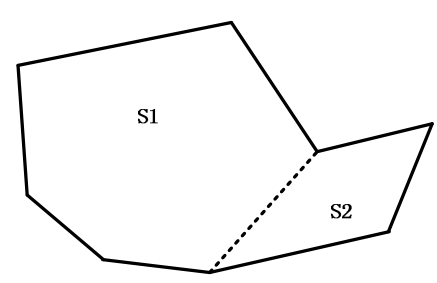

Fig. 4 Divide non-convex polygon into convex

The "same side judgment" is present in this paper. In Fig.3, if inside the convex polygon, the vehicle is always in the left of each counter-clockwise edge (right of each clockwise edge). Assuming " $\mathrm{M}$ " $(x, y)$, the " $\mathrm{d}$ " (distance from " $\mathrm{M}$ " to the vertices $p_{k-1}\left(x_{k-1}, y_{k-1}\right)$ and $p_{k}\left(x_{k}, y_{k}\right)$ is followed in Eq. 7:

$$
d=\frac{\left(y_{k}-y_{k-1}\right) x+\left(x_{k-1}-x_{k}\right) y+y_{k-1} x_{k}-x_{k-1} y_{k}}{\sqrt{\left(y_{k}-y_{k-1}\right)^{2}}+\left(x_{k-1}-x_{k}\right)^{2}} .
$$

In Eq. 7, "d" is signed. While seeing form the above vertice $P_{k-1}$ to the next $\boldsymbol{P}_{k-1}$, if "M" in the left of the mission track, the $\mathrm{d}$ is positive, otherwise, is negative.

In briefly, the "same side judgment" is: each distance from "M" to each edge is positive or negative in the same time, the " $\mathrm{M}$ " is inside, otherwise, is outside.

For the non-convex polygon area, divide it into 2 or more convex polygons to judge, as shown in Fig. 4. If inside one part, it can be judged inside the whole area.

In that way, the vehicle whether in the yard can be checked only by GPS location

\section{Application}

The research result is applied in an unmanned iron ore yard. The north area and the south area are disconnected. Each yard has a stacker crane track in the middle. The GPS application unit sent the location message to the monitoring center while entering the yard. The center monitors and manages the yard unmanned, avoiding the crash between the cars and crane. The system also suit for cargo traffic monitor.

The Human-Machine Interface (HMI) of the system is shown in Fig. 5. It contains two parts: serial communication part and yard map part. 
User can select the port and Baud rate to build connection in the serial communication part. The latest indicate prompts and commands are shown in the prompt box in sequence. User can sent custom information to the terminal on the cars through Digital transmission module manually.

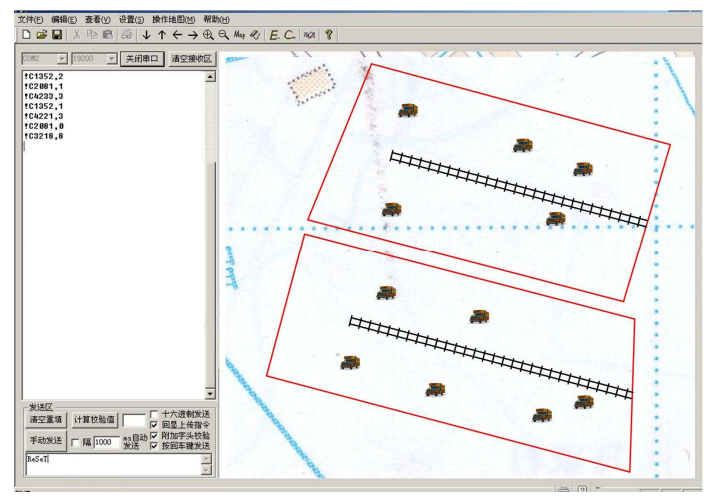

Fig. 5 Monitor system for a iron ore yard

The yard map can show the car's location in the map. Users can select show each vehicle's traveling track or not show. Related operations can be achieved through the Up, Down, Left, Right, Zoom in, Zoom out, Recovery buttons in the toolbar. When overlap, click the Eraser to clean the previous tracks.

The reliable operation of designed GPS applicable unit and monitor system shows the research result is right and stable.

\section{Conclusion}

For the vehicle manage require of the large materials yard, this paper research the monitor problem based on u- Blox GPS. Design the GPS applicable unit, research the Setting Method of LEA-4H and the extract skill of GPS Protocol data. Research the Grid-based map technology to mark, zoom, translation the map to match the vehicle track.

1) Based on u- Blox GPS, the GPS applicable unit has been designed in this paper.

2) The "same side judgment" has been proposed to check the vehicle whether in the yard only by GPS location.

The research result has been proved correct and stable through the reliable operation of an unmanned iron ore yard for years. It can provide good reference for the other material yard and fleet's monitor and manage system's design.

\section{References}

[1] Liu jiyu,"The GPS satellite navigation and positioning principle and method"'[M]. Science press. Beijing. 2003.8

[2] Gong zhenchun, "GPS in the micro uav navigation and positioning of the research and application" [D]. Master thesis, zhejiang university. 2005.1

[3] http://www.ublox.com/

[4] Qu lei and Liu shengde and Hu xianbin, "The ZigBee technology and application"[M]. Beijing university of aeronautics and astronautics press. Beijing.2007.9

[5] Klaus Betke. NMEA 0183. May,2000, revised Aug. 2001. www.nmea.org

[6] u-Blox company. ANTARIS Protocol Specification. 2007 